\title{
Diversity or disarray? A systematic review of decision-making capacity for treatment and research in schizophrenia and other non-affective psychoses
}

\author{
B. W. J. Spencer ${ }^{1,2 *}$, G. Shields ${ }^{2}$, T. Gergel ${ }^{1}$, M. Hotopf ${ }^{1,2}$ and G. S. Owen ${ }^{1,2}$ \\ ${ }^{1}$ King's College London, Mental Health, Ethics and Law Research Group, Department of Psychological Medicine, Institute of Psychiatry, Psychology \\ and Neuroscience, Weston Education Centre, 10 Cutcombe Road, London, SE5 9RJ, UK \\ ${ }^{2}$ South London and Maudsley NHS Foundation Trust, Maudsley Hospital, Denmark Hill, London, SE5 8AZ, UK
}

Background. Valid consent for treatment or research participation requires that an individual has decision-making capacity (DMC), which is the ability to make a specific decision. There is evidence that the psychopathology of schizophrenia can compromise DMC. The objective of this review was to examine the presence or absence of DMC in schizophrenia and the socio-demographic/psychopathological factors associated.

Methods. We searched three databases Embase, Ovid MEDLINE(R), and PsycINFO for studies reporting data on the proportion of DMC for treatment and research (DMC-T and DMC-R), and/or socio-demographic/psychopathological associations with ability to make such decisions, in people with schizophrenia and related illnesses.

Results. A total of 40 studies were identified. While high levels of heterogeneity limited direct comparison, meta-analysis of inpatient data showed that DMC-T was present in $48 \%$ of people. Insight was strongly associated with DMCT. Neurocognitive deficits were strongly associated with lack of DMC-R and to a lesser extent DMC-T. With the exception of years of education, there was no evidence for an association with socio-demographic factors.

Conclusions. Insight and neurocognitive deficits are most closely associated with DMC in schizophrenia. The lack of an association with socio-demographic factors dispels common misperceptions regarding DMC and characteristics such as age. Although our results reveal a wide spectrum of DMC-T and DMC-R in schizophrenia, this could be partly due to the complexity of the DMC construct and the heterogeneity of existing studies. To facilitate systematic review research, there is a need for improvement within research study design and increased consistency of concepts and tools.

Received 11 October 2016; Revised 2 February 2017; Accepted 2 February 2017; First published online 26 April 2017

Key words: Competence, decision-making capacity, informed consent, mental capacity, psychosis, schizophrenia.

\section{Introduction}

Consent for treatment or research requires the individual to have the ability to make a decision, known as decision-making capacity (DMC) (Grisso \& Appelbaum, 1998). Many legislative regions now use DMC to regulate treatment or research participation (Appelbaum, 2007; Nicholson et al. 2008).

Non-affective psychotic illnesses such as schizophrenia and its symptoms can compromise DMC (Appelbaum \& Grisso, 1995; Grisso \& Appelbaum, 1995; Grisso et al. 1995). Assessments of DMC for treatment (DMC-T) can result in substantial changes in a person's experience of treatment: either autonomous

\footnotetext{
* Address for corresponding author: Dr B. W. J. Spencer, King's College London, Mental Health, Ethics and Law Research Group, Department of Psychological Medicine, Institute of Psychiatry, Psychology and Neuroscience, Weston Education Centre, 10 Cutcombe Road, London, SE5 9RJ, UK.

(Email: Benjamin.spencer@kcl.ac.uk)
}

decision making for oneself or decisions made by others. In decisions regarding research participation lacking DMC for research (DMC-R), or being deemed likely to lack it, may lead to ineligibility for research recruitment.

Given that DMC is decision-specific, the information to be understood is different for each decision (Jacob et al. 2013). Therefore, the same individual may lack DMC for one decision but not another (Grisso \& Appelbaum, 1998). Furthermore, DMC also involves considering this information within the context of personal circumstances, beliefs, and values. Understanding lack of DMC in people with schizophrenia, the associated symptoms, to what extent loss is decisionspecific and how individual context might effect DMC is of critical importance to all clinicians working with this mental disorder.

DMC is a complex construct. The underlying abilities, e.g. understanding or reasoning, can be measured as dimensional or categorical (such as by applying a cut-off). In clinical and legal practice a decision must

This is an Open Access article, distributed under the terms of the Creative Commons Attribution licence (http://creative commons.org/licenses/by/4.0/), which permits unrestricted reuse, distribution, and reproduction in any medium, provided the original work is properly cited. 
be made that the person has, or lacks, the ability for $\mathrm{DMC}$, making it a binary judgement.

Different legislative regions have separate legal definitions for the abilities which are jointly necessary for DMC. In England and Wales the legal test is defined by the Mental Capacity Act 2005 (MCA) and requires the ability to: 'understand' the information relevant to a decision; 'retain' it; 'use or weigh' the information to arrive at a decision; and 'communicate' that decision. Many US states use a similar model - the 'four factor model' of 'understanding', 'appreciation', 'reasoning', and 'expressing a choice' (Appelbaum \& Grisso, 1995; Grisso \& Appelbaum, 1995; Grisso et al. 1995). The four factors of the MCA are viewed as largely synonymous with the US four factors, with 'use or weigh' incorporating 'appreciation' and 'reasoning' (Owen et al. 2009a). Assessments of DMC for legal and medical consent are made by clinicians or the court based on the relevant legal test. Such assessments are, ultimately, the 'gold standard' of DMC assessment and, although the court is the final arbitrator, the assessment process itself is delegated mainly to clinicians.

Research into DMC has therefore measured DMC in one of three ways:

(1) 'Dimensional scores': use of structured tools to psychometrically assess performance within individual domains of abilities deemed core to DMC (such as the 'four factor model') to return a score for each dimension.

(2) 'Cut-off standard': applying a cut-off or scoring algorithm to 'dimensional scores'.

(3) 'Judgement standard': clinical or court assessment of DMC returning a binary judgement. This may or may not be guided by legal criteria and dimensions to be assessed (such as the MCA in the UK or the 'four factor model' in the USA).

Each approach has both advantages and limitations: The 'cut-off standard' and 'dimensional scores' are primarily for research use, and allow for a more detailed exploration of symptoms contributing to DMC vulnerability than the 'judgement standard' permits. The 'judgement standard' is the standard of DMC in clinical and legal practice, although it may be guided by the other two tools. A highly influential study, the MacArthur Treatment Competence study (Appelbaum \& Grisso, 1995; Grisso \& Appelbaum, 1995; Grisso et al. 1995), developed a set of tools for assessing DMC-T using 'dimensional scores' based on the 'four factor model'. These were subsequently condensed into the MacArthur Competence Assessment Tool for Treatment (MacCAT-T) (Grisso et al. 1997) and then adapted for decisions regarding Clinical Research (MacCAT-CR) (Appelbaum \&
Grisso, 2001). These tools led to an explosion of research into DMC, with many studies measuring DMC using 'dimensional scores'.

The objective of the present review was to explore proportions and clinical associations of DMC in people with schizophrenia using these three standards (for the purpose of clarity we use the term 'schizophrenia' to refer to non-affective psychosis including, but not limited to, schizoaffective disorder, delusional disorder, transient psychotic episodes etc.). Our research questions were:

(1) What proportion of people with schizohrenia has DMC for specified civil decisions (such as treatment or participation in research) in specified settings (e.g. inpatient, outpatient)?

(2) What are the associations with DMC for civil decisions? We pre-specified associations of interest as positive symptoms, negative symptoms, general symptoms of psychosis, neurocognitive symptoms, affective symptoms, awareness of illness (insight) and socio-demographic variables (age, sex, ethnicity and educational level).

To our knowledge there have been two previous systematic reviews into DMC in schizophrenia, rather than in conjunction with other diagnoses such as dementia or bipolar affective disorder (Jeste et al. 2006; Wang et al. 2016). However, unlike ours, both these reviews focused primarily on a comparing dimensional DMC scores in those diagnosed with schizophrenia and in 'normal controls', finding that people with schizophrenia did less well.

\section{Methods}

\section{Eligibility criteria}

We included studies published in English, which assessed the DMC of samples of people over the age of 18 diagnosed with non-affective psychosis, as defined by: f20-29 ICD-10 (World Health Organization., 1993) or 295, 297, 298 DSM-IV (American Psychiatric Association., 1994). We included studies measuring DMC or domains of DMC using the three approaches described above: the 'judgement standard'; 'cut-off standard'; or 'dimensional scores'. We excluded non-civil assessments of DMC (such as fitness to plead).

\section{Search}

We used OVID to search Embase, Ovid MEDLINE (R), and PsycINFO. Our search string was chosen following several trial iterations of searches to maximise the sensitivity of the search, given that 'capacity' has multiple homonyms. Our final search string was a title and 
abstract search of: (capacity or competence or competency or 'decision making' or 'decision-making') AND (schizophrenia or psychosis or 'mental illness' or 'mental disorder' or psychotic). The search was completed on 16 February 2015, with results exported to Endnote X7. The citation search was performed on 17 July 2015, with all steps in both searches performed by B Spencer (BS).

References reporting data from the same study were excluded unless the samples were mutually exclusive. Exclusion occurred at the data extraction stage and following correspondence with the authors. In these cases the reference best suited to the review was selected by BS for retention within the final selection. In addition, if multiple references reported complementary analyses of the same sample they were treated as one reference in the final analysis.

\section{Data collection and data items}

BS extracted all data using a data extraction form which specified: population studied and associated demographics; nature of decision for which DMC was assessed (whether it was for a decision related to the present disorder, such as treatment for schizophrenia rather than treatment for another unrelated medical condition, and, in the case of DMC-R, whether it was for hypothetical or real study involvement); outcome of the DMC assessment (proportions from studies using 'judgement standard' and 'cut-off standard'); effect sizes (ES) for any associations between DMC and variables of interest. Only summary data, rather than data on individual items of tools were extracted from studies. The only exception was item G12 on the Positive and Negative Syndrome Scale (PANSS) (Kay et al. 1987) 'lack of judgement and insight', which we chose to include, given that this was the primary measure of insight used in several studies.

\section{Statistical analysis}

Confidence intervals (95\%) were calculated for proportions of DMC following 'judgement standard' or 'cutoff standard' using the Wilson score interval. Odds ratios and correlation coefficients were converted into ES for our principal summary measure. Given that some studies were able to detect very small ES, we modified the Cohen criteria (Cohen, 1992) to: $>0$ to $\leqslant 0.3$ small ES, $\geqslant 0.3$ medium ES, and $\geqslant 0.5$ large ES.

We aimed to meta-analyse the proportions of people with DMC as measured by the 'judgement standard'. For studies to be eligible for the meta-analysis for $\mathrm{DMC}$, they needed to test DMC for similar decisions (e.g. DMC-T for treatment of the present disorder) within a homogenous setting (e.g. solely inpatients or outpatients) and without other factors likely to bias the result as decided by the authors (e.g. not systematically excluding detained or severely unwell people). Meta-analysis of proportions was performed using STATA 14 (StataCorp). Given the residual heterogeneity between studies, a random effects model was used.

\section{Risk of bias assessment}

To our knowledge there has been no prior attempt to appraise quality in DMC studies. We considered certain factors to be important based on our clinical experience when reviewing studies on DMC. These included: (1) the exact nature of the decision for which DMC is being assessed (whether it was real, hypothetical, related to the present disorder - schizophrenia or wholly unrelated), as this may impact on effect of symptoms of schizophrenia on DMC (for example, whether insight into illness is relevant to the decision, whether the decision was cognitively demanding, etc.); (2) homogenous setting of recruitment (either all inpatients or outpatients and thus controlling for hidden confounders in these settings); (3) ability to recruit people with a range of severity of illness within a specified setting, given that this would likely impact on DMC (e.g. were people deemed to be 'too unwell' systematically excluded from the sample). We developed a risk of bias assessment based on these which demonstrated critical risk of bias for the majority of studies (available from the authors on request). As we wanted to provide an overview of the literature, we decided to exclude a risk of bias assessment from this review, but comment further on the quality of research in the discussion.

\section{Results}

Of 11658 references screened from titles, 682 references went to full text review, and 40 met our inclusion criteria (see Fig. 1) (Weinstock et al. 1984; Veliz \& James, 1987; Norko et al. 1990; Bean et al. 1994; Appelbaum \& Grisso, 1995; Grisso \& Appelbaum, 1995; Grisso et al. 1995, 1997; Carpenter et al. 2000; Moser et al. 2002, 2005, 2006; Valletto et al. 2002; Wong et al. 2000, 2005; Bellhouse et al. 2003; Kovnick et al. 2003; Vollmann et al. 2003; Cohen et al. 2004; Palmer et al. 2004, 2005; Srebnik et al. 2004; Cairns et al. 2005; Eyler et al. 2005, 2007; Koren et al. 2005; Stroup et al. 2005; Candilis et al. 2006, 2008; Palmer \& Jeste, 2006; Dunn et al. 2007; Moye et al. 2008; Kavanagh et al. 2008; Jeste et al. 2009; Owen et al. 2009a, 2011; De Marco et al. 2010; Capdevielle et al. 2009; Linder et al. 2012; Di \& Cheng, 2013; Fischer et al. 2013; Kumar et al. 2013; Lan et al. 2013; Raffard et al. 2013; Skipworth et al. 2013; Chiu et al. 2014). A 


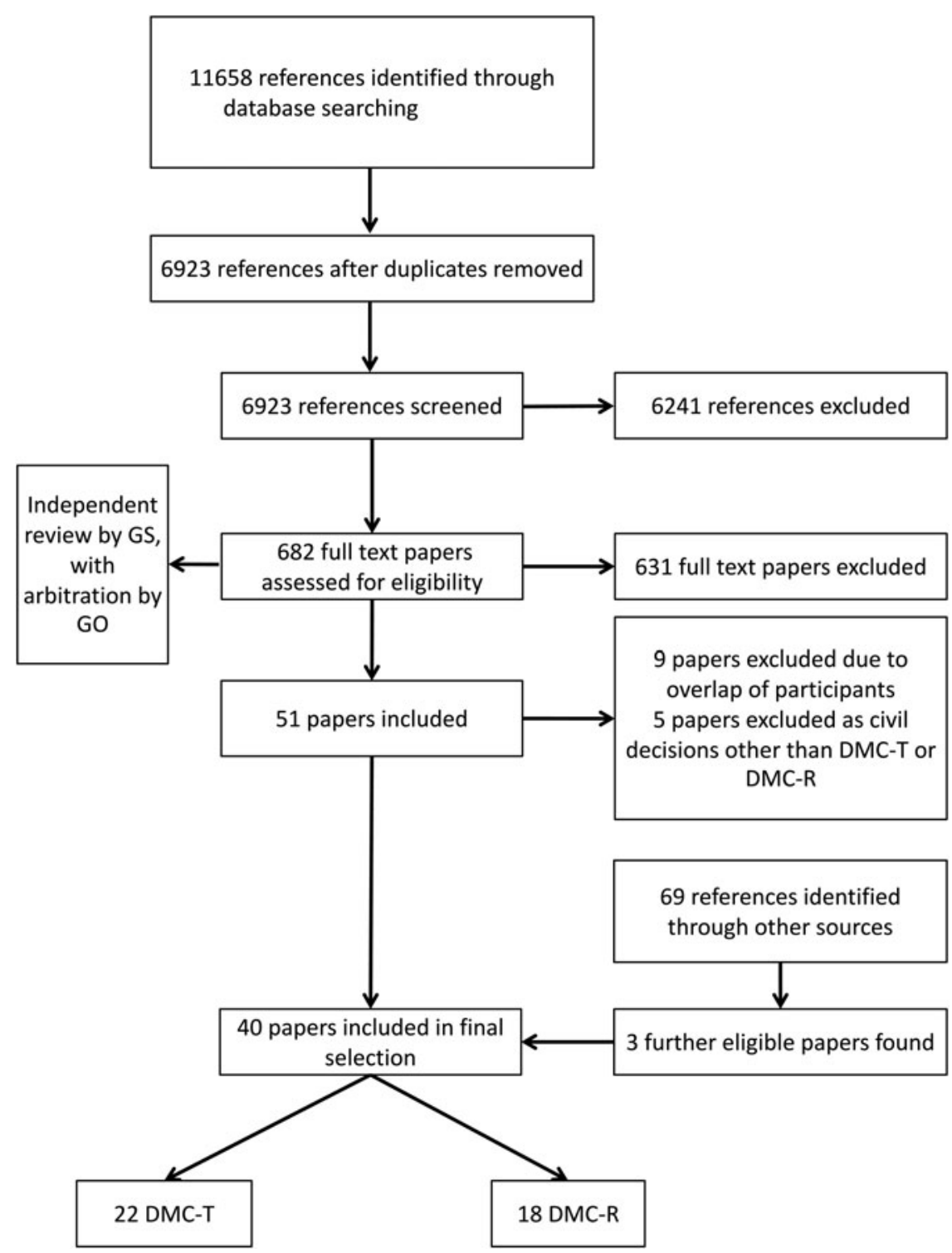

Fig. 1. Flow diagram.

clinician with expertise in the field [G Shields (GS)] performed an independent review of all 682 references applying the inclusion and exclusion criteria. Inter-rater reliability between BS and GS was high ( $K$ $=0.80$ ). Disagreements were resolved following discussion between BS and GS, while any unresolved disputes went to G Owen (GO) as final arbiter $(n=3)$.

Heterogeneity between studies was high, with considerable variation in study design, population, measurements and the nature of decision for which DMC was assessed (see Table 1). Many studies reported only partial data for the outcomes of interest, while the studies assessing DMC using a 'judgement standard' rarely presented any associations with our prespecified variables of interest. Results from all studies and characteristics are available in the online supplemental data table. Most studies assessed psychopathology using either the PANSS or Brief Psychiatric Rating Scale (BPRS) (Overall \& Gorham, 1962). Many studies used a range of diverse individual neurocognitive sub-tests from various test batteries (such as the Wechsler Adult Intelligence Scale - III [WAIS-III (Wechsler, 1997)] without a summary score provided. These individual results were not extracted, given the difficulties in direct comparison between studies.

Given the limited numbers of studies investigating decisions other than DMC-T and DMC-R $(n=5)$, we limited our review to treatment and research $(n=40)$. These five studies considered DMC for organ donation (De Marco et al. 2010), making a psychiatric advance directive (Valletto et al. 2002; Srebnik et al. 2004; 
Table 1. Main characteristics of studies

\begin{tabular}{|c|c|c|c|c|c|c|c|c|}
\hline $\begin{array}{l}\text { DMC-T } \\
\text { or } \\
\text { DMC-R }\end{array}$ & $\begin{array}{l}\text { Standard } \\
\text { used }\end{array}$ & $\begin{array}{l}\text { Decision } \\
\text { related to } \\
\text { schizophrenia }\end{array}$ & $\begin{array}{l}\text { Population } \\
\text { setting }\end{array}$ & $\begin{array}{l}\text { Decision to be made by } \\
\text { participant }\end{array}$ & Format of assessment & Associations reported & Other features & Title \\
\hline \multirow[t]{9}{*}{ DMC-T } & \multirow[t]{9}{*}{$\begin{array}{l}\text { Expert } \\
\text { judgement }\end{array}$} & \multirow[t]{7}{*}{ Related } & Mixed & $\begin{array}{l}\text { Current psychiatric } \\
\text { treatment with medication }\end{array}$ & $\begin{array}{l}\text { Clinical assessment under the } \\
\text { criteria of the MCA } 2005 \text { and } \\
\text { structured using the MacCAT-T }\end{array}$ & & $\begin{array}{l}\text { Mixed inpatients and } \\
\text { outpatients under forensic } \\
\text { services }\end{array}$ & $\begin{array}{l}\text { Skipworth et al. } \\
\text { (2013) }\end{array}$ \\
\hline & & & \multirow[t]{6}{*}{ Inpatients } & \multirow[t]{2}{*}{$\begin{array}{l}\text { Treatment in hospital with } \\
\text { ECT }\end{array}$} & $\begin{array}{l}\text { Clinical assessment (no further } \\
\text { details) }\end{array}$ & & Inpatients requiring $\mathrm{ECT}$ & $\begin{array}{l}\text { Bean et al. } \\
\text { (1994) }\end{array}$ \\
\hline & & & & & $\begin{array}{l}\text { Clinical assessment (no further } \\
\text { details) }\end{array}$ & & $\begin{array}{l}\text { Patients who received ECT } \\
\text { without consent }\end{array}$ & $\begin{array}{l}\text { Chiu et al. } \\
\text { (2014) }\end{array}$ \\
\hline & & & & $\begin{array}{l}\text { Current psychiatric } \\
\text { treatment }\end{array}$ & $\begin{array}{l}\text { Clinical assessment (no further } \\
\text { details) }\end{array}$ & & $\begin{array}{l}\text { Referred to the Court for } \\
\text { determination of lack of } \\
\text { competency to refuse or } \\
\text { consent to treatment } \\
\text { forensic population }\end{array}$ & $\begin{array}{l}\text { Veliz \& James } \\
\text { (1987) }\end{array}$ \\
\hline & & & & $\begin{array}{l}\text { Current admission and } \\
\text { psychiatric treatment }\end{array}$ & $\begin{array}{l}\text { Clinical assessment under the } \\
\text { criteria of the legal precursor to } \\
\text { the MCA and structured } \\
\text { according to this }\end{array}$ & & & $\begin{array}{l}\text { Bellhouse et al. } \\
\text { (2003) }\end{array}$ \\
\hline & & & & $\begin{array}{l}\text { Current psychiatric } \\
\text { treatment with medication }\end{array}$ & $\begin{array}{l}\text { Clinical assessment under the } \\
\text { criteria of the legal precursor to } \\
\text { the MCA and structured and } \\
\text { structured using the MacCAT-T }\end{array}$ & & & $\begin{array}{l}\text { Cairns et al. } \\
(2005)\end{array}$ \\
\hline & & & & $\begin{array}{l}\text { Either current admission or } \\
\text { psychiatric treatment in } \\
\text { hospital }\end{array}$ & $\begin{array}{l}\text { Clinical assessment under the } \\
\text { criteria of the MCA and } \\
\text { structured using the MacCAT-T }\end{array}$ & SAI, BPRS & & $\begin{array}{l}\text { Owen et al. } \\
(2009 a, 2011)\end{array}$ \\
\hline & & Unrelated & N/A & $\begin{array}{l}\text { Physical health treatment in } \\
\text { a medical hospital }\end{array}$ & $\begin{array}{l}\text { Clinical assessment using criteria } \\
\text { based on early precursors to the } \\
\text { four factor model, unstructured }\end{array}$ & & $\begin{array}{l}\text { Medically unwell in a } \\
\text { physical health hospital } \\
\text { referred for determination } \\
\text { of DMC-T for medical } \\
\text { treatment. }\end{array}$ & $\begin{array}{l}\text { Weinstock et al. } \\
\text { (1984) }\end{array}$ \\
\hline & & Unclear & Mixed & Routine blood test & $\begin{array}{l}\text { Clinical assessment under the } \\
\text { criteria of the legal precursor to } \\
\text { the MCA and structured }\end{array}$ & & $\begin{array}{l}\text { Decision for a blood test - } \\
\text { unclear degree related. }\end{array}$ & $\begin{array}{l}\text { Wong et al. } \\
(2000)\end{array}$ \\
\hline
\end{tabular}




\begin{tabular}{|c|c|c|c|c|}
\hline \multirow[t]{3}{*}{ Cut-off } & Related & Inpatients & $\begin{array}{l}\text { Current psychiatric } \\
\text { treatment }\end{array}$ & SSICA \\
\hline & & & $\begin{array}{c}\text { Current admission and } \\
\text { psychiatric treatment }\end{array}$ & $\begin{array}{l}\text { Tool assessing early precursors } \\
\text { to the four factor model }\end{array}$ \\
\hline & Unrelated & Outpatients & $\begin{array}{l}\text { Hypothetical medical } \\
\text { vignette involving a toe } \\
\text { amputation or femoral } \\
\text { bypass in non-healing toe } \\
\text { ulcer }\end{array}$ & ACCT \\
\hline \multirow[t]{4}{*}{$\begin{array}{l}\text { Four factor } \\
\text { scores }\end{array}$} & Related & Outpatients & $\begin{array}{l}\text { Current psychiatric } \\
\text { treatment with atypical } \\
\text { antipsychotic medication }\end{array}$ & MacCAT-T \\
\hline & & & $\begin{array}{l}\text { Current psychiatric } \\
\text { treatment }\end{array}$ & MacCAT-T. \\
\hline & & & $\begin{array}{l}\text { Current psychiatric } \\
\text { treatment with } \\
\text { antipsychotic medication }\end{array}$ & MacCAT-T \\
\hline & & Inpatients & $\begin{array}{l}\text { Current psychiatric } \\
\text { treatment }\end{array}$ & MacCAT-T precursors \\
\hline
\end{tabular}

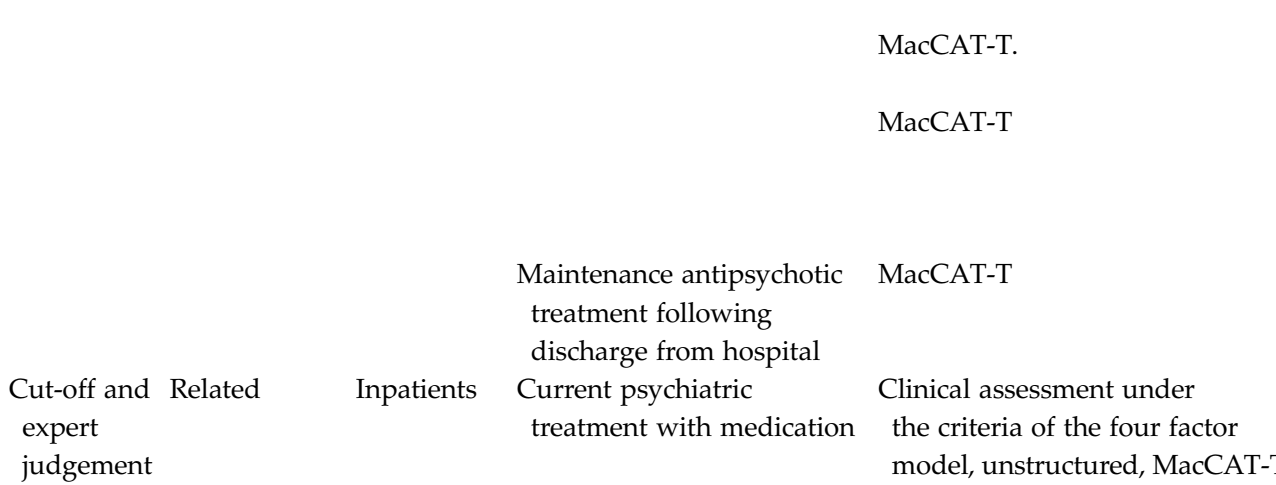

Socio-demographics Guardian also needed to agree Di \& Cheng in order to participate in (2013) study.

No detained patients Norko et al. (1990)

$\geqslant 60$ years old $\quad$ Moye $e t$ al. (2008)

PANSS, BPRS, DRS, Outpatients, although most Palmer et al. socio-demographics. living at community assisted (2004) living facilities. $\geqslant 40$ years old

SUMD, PANSS, BDI, No treatment changes for the Capdevielle socio-demographics past month (2009) PANSS, BDI, No treatment changes for the Raffard et al. socio-demographics past month (2013)

BPRS, VCF, Clinicians requested really

socio-demographics

unwell people to not be

Appelbaum \& Grisso (1995); recruited.

BRPS,

socio-demographics

Grisso \&

Appelbaum

(1995); Grisso

et al. (1995)

Grisso et al.

(1997)

Within 2 weeks of admission Koren et al. when clinician has (2005)

determined them able to

cooperate

G12 PANSS insight, Before discharge from hospital Wong et al.

PANSS, MADRS,

socio-demographic

Socio-demographics

No detained patients

Vollmann et al. (2003) 


\begin{tabular}{|c|c|c|c|c|c|}
\hline $\begin{array}{l}\text { DMC-T } \\
\text { or } \\
\text { DMC-R }\end{array}$ & $\begin{array}{l}\text { Standard } \\
\text { used }\end{array}$ & $\begin{array}{l}\text { Decision } \\
\text { related to } \\
\text { schizophrenia }\end{array}$ & $\begin{array}{l}\text { Population } \\
\text { setting }\end{array}$ & $\begin{array}{l}\text { Decision to be made by } \\
\text { participant }\end{array}$ & Format of assess \\
\hline \multirow[t]{5}{*}{ DMC-R } & \multirow[t]{2}{*}{ Cut-off } & \multirow[t]{2}{*}{ Related } & Outpatients & $\begin{array}{l}\text { RCT of adjunctive therapy } \\
\text { to usual antipsychotic } \\
\text { regimen }\end{array}$ & mESC \\
\hline & & & Mixed & $\begin{array}{l}\text { RCT of atypical } \\
\text { antipsychotic medication }\end{array}$ & MacCAT-CR \\
\hline & \multirow[t]{3}{*}{$\begin{array}{l}\text { Four factor } \\
\text { scores }\end{array}$} & \multirow[t]{3}{*}{ Related } & Outpatients & $\begin{array}{l}\text { fMRI study of } \\
\text { decision-making capacity }\end{array}$ & MacCAT-CR \\
\hline & & & \multirow[t]{2}{*}{ Mixed } & $\begin{array}{l}\text { RCT of antipsychotic } \\
\text { medication }\end{array}$ & MacCAT-CR \\
\hline & & & & $\begin{array}{l}\text { CATIE study (naturalistic } \\
\text { antipsychotic treatment } \\
\text { study) }\end{array}$ & MacCAT-CR \\
\hline
\end{tabular}

Observational study of

tardive dyskinesia and other side effects of atypical antipsychotic medications

Inpatients RCT of antipsychotic medication

Unclear

Outpatients RCT of cognitive enhancement medication
MacCAT-CR

MacCAT-CR

MacCAT-CR

MacCAT-CR

MacCAT-CR

Associations reported Other features

Title

BPRS, MMSE

\section{DMC-R)}

BIQ, PANSS, HAM-D, Mixed outpatient and neurocognitive $Z$

score, DRS,

socio-demographics

PANSS,

neurocognitive $Z$

score,

socio-demographics inpatients, including board and care homes. Aged $\geqslant 50$

Outpatient study recruiting from board and care homes

Carpen

Mixed inpatients and outpatients already recruited (2005) to the CATIE study (having suboptimal antipsychotic treatment) and passing a

MacCAT-CR based DMC-R threshold $(U \geqslant 16)$

BIQ, PANSS, HAM-D, Mixed inpatients and neurocognitive $\mathrm{Z}$ outpatients, some in board score, outpatients, some in board
and care homes. Aged $\geqslant 40$

socio-demographics BPRS, VCF

Long stay patients on a research ward with schizophrenia.

PANSS, MMSE, Aged $\geqslant 60$

socio-demographics

Recruited before medication free period as an inpatient $f$ treatment of schizophrenia.

Only data on correlations are the effect of interventions

Only data on correlations are Moser et al. the effect of interventions
Palmer \& Jeste (2006)

Kovnick et al.

Palmer et al. (2005)

Moser et al. (2005) (2006) 


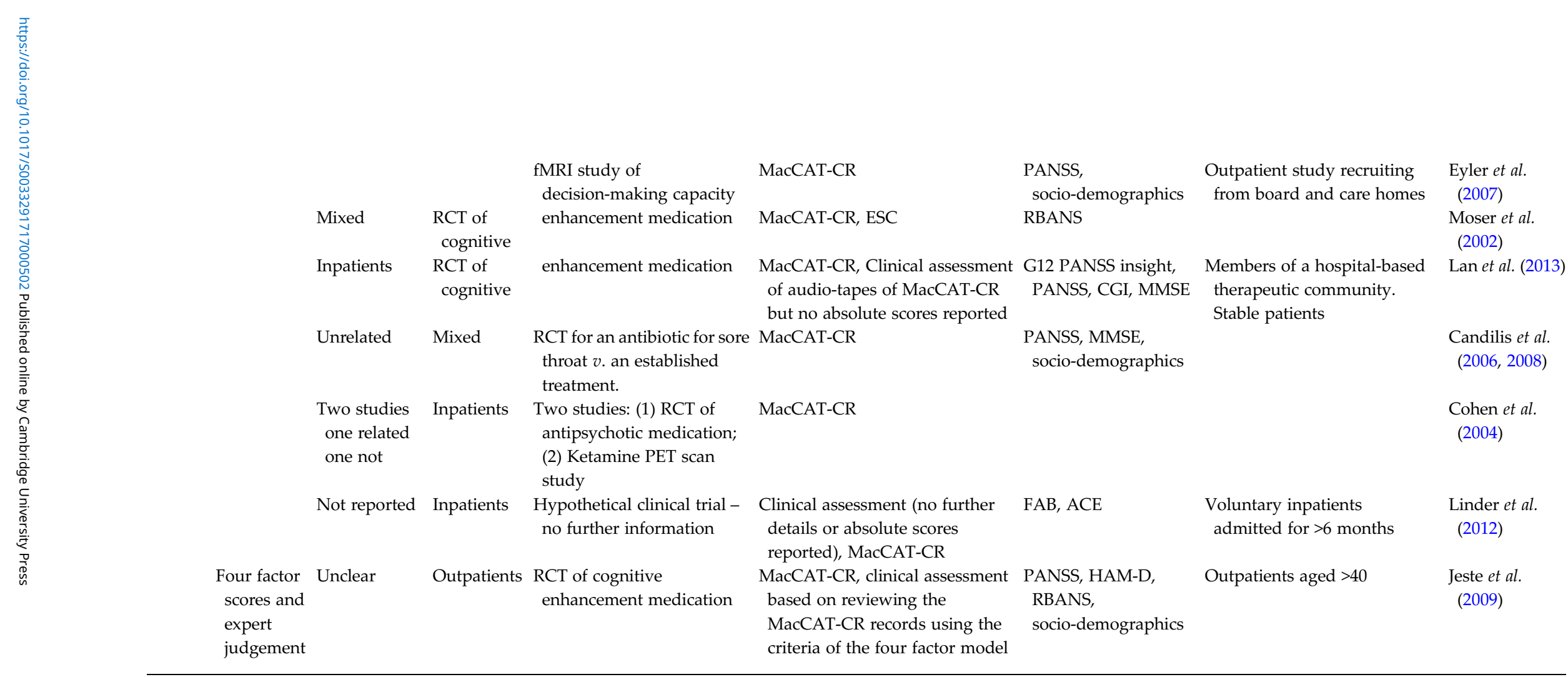

Legend: ACCT, Assessment of Capacity to Consent to Treatment Interview, ACE, Addenbrooke's Cognitive Exam, BDI, Beck Depression Inventory, BIQ, Birchwood Insight Questionnaire, BPRS, Brief Psychiatric Rating Scale, CGI, Clinical Global Impression, DRS, Mattis Dementia Rating Scale, ESC, Evaluation to Sign Consent, FAB, Frontal Assessment Battery, HAM-D, Hamilton Depression Rating Scale, MacCAT-CR, MacArthur Competence Assessment Tool for Clinical Research, MacCAT-T, MacArthur Competence Assessment Tool for Treatment, MADRS, Montgomery-Åsberg Depression Rating Scale, mESC, Modified Evaluation to Sign Consent, MMSE, Mini-Mental State Exam, PANSS, Positive and Negative Syndrome Scale, RBANS, Repeatable Battery for the Assessment of Neuropsychological Status, SAI, Schedule for the Assessment of Insight, SSICA, Semi-structured inventory for competence assessment, SUMD, Scale to assess Unawareness of Mental Disorder, VCF, Verbal Cognitive Functioning.

NB - many studies also reported on individual neurocognitive sub-tests from various test batteries, these are not presented in this table. 
Kumar et al. 2013), and DMC to manage one's own finances (Barrett et al. 2009).

\section{Performance on different standards of DMC}

Proportion of DMC-T in studies using 'judgement standard' and 'cut-off standard'

Ten studies reported the proportion of DMC-T amongst participants when using the judgement standard' (Weinstock et al. 1984; Veliz \& James, 1987; Bean et al. 1994; Wong et al. 2000; Bellhouse et al. 2003; Vollmann et al. 2003; Cairns et al. 2005; Owen et al. 2009a, 2011; Skipworth et al. 2013; Chiu et al. 2014), while three studies used the 'cut-off standard' (Norko et al. 1990; Moye et al. 2008; Di \& Cheng, 2013). Characteristics and results from all studies providing data on 'judgement standard' or 'cut-off standard' of assessment are presented in Table 2 (Chiu et al. 2014 and Norko et al. 1990 are excluded and considered separately below). The range of proportions of DMC-T reported by all studies is large (11-100\%) and there is significant heterogeneity between studies: six studies recruited from inpatient settings (Veliz \& James, 1987; Bean et al. 1994; Bellhouse et al. 2003; Vollmann et al. 2003; Cairns et al. 2005; Owen et al. 2009a, 2011; Di \& Cheng, 2013), one from outpatients (Moye et al. 2008), two from mixed inpatients and outpatient settings (Wong et al. 2000; Skipworth et al. 2013), and one from a general medical hospital setting (Weinstock et al. 1984). Seven studies assessed DMC-T for a decision that was related to the disorder (hospital admission or treatment for schizophrenia) (Veliz \& James, 1987; Bean et al. 1994; Bellhouse et al. 2003; Vollmann et al. 2003; Cairns et al. 2005; Owen et al. 2009a, 2011; Di \& Cheng, 2013; Skipworth et al. 2013); two assessed DMC-T for medical treatment unrelated to schizophrenia (Weinstock et al. 1984; Moye et al. 2008); and one assessed DMC-T for treatment with an unclear relationship to schizophrenia (Wong et al. 2000). Two studies assessed DMC-T as a naturalistic study in which people were recruited following concerns regarding a lack of DMC-T having been raised (Weinstock et al. 1984; Veliz \& James, 1987).

It was only within the set of studies recruiting from inpatient settings that there were two or more studies sufficiently comparable with each other in terms of recruitment setting and nature of decision for which DMC-T was assessed in order to be eligible to undergo meta-analysis (Bean et al. 1994; Bellhouse et al. 2003; Cairns et al. 2005; Owen et al. 2009a, 2011; Di \& Cheng, 2013). These studies assessed DMC-T for psychiatric admission and/or treatment in hospital with medication or ECT; three were UK-based and used the MCA legal standard. The range of people with DMC-T was $26-67 \%$. A meta-analysis of proportions using a random effects model indicated high heterogeneity $\left(I^{2}-84.41 \%\right)$ and a pooled proportion of $48 \%$ (95\% CI 29-66\%) with DMC-T (see Fig. 2).

Of the two studies considered separately: Norko et al. (1990), used a range of 'cut-offs' based on combinations of 'dimensional scores', and found that DMC varied between $45 \%$ and $80 \%$, depending on the precise cut-off used. Chiu et al. (2014) reported the characteristics of people given Electro-Convulsive Therapy (ECT) without consent, dichotomising the groups into people without DMC-T given ECT and people with DMC-T given ECT despite objecting. In those having ECT without consent, $n=13,76 \%$ (95\% CI $53-$ 90\%) lacked DMC-T.

\section{Proportion of DMC-R from 'judgement standard' and 'cut-off standard'}

One study (Dunn et al. 2007) tested DMC-R concerning a hypothetical decision related to schizophrenia in a mixed population of inpatients and outpatients. It used three 'cut-off standards', 'least'; 'intermediate'; and 'most', (the 'Dunn standard') and found that 92, $81,43 \%$ met their standards for each of these, respectively. Another study used a 'judgement standard' to test DMC-R amongst older outpatients (Jeste et al. 2009) and found that $47 \%$ of those undergoing 'routine consent' had DMC-R.

\section{'Dimensional scores' and DMC-T/DMC-R}

Five studies reported 'dimensional scores' from MacCAT-T sub-scales (Grisso et al. 1997; Palmer et al. 2004; Koren et al. 2005; Wong et al. 2005; Capdevielle et al. 2009), and thirteen studies reported 'dimensional scores' from MacCAT-CR sub-scales (Carpenter et al. 2000; Moser et al. 2002, 2005, 2006; Kovnick et al. 2003; Palmer et al. 2005; Stroup et al. 2005; Candilis et al. 2006; Palmer \& Jeste, 2006; Dunn et al. 2007; Eyler et al. 2007; Candilis et al. 2008; Jeste et al. 2009; Lan et al. 2013). These were all reported as arithmetic means and standard deviations. One study provided 'dimensional scores' from the precursor tools to the MacCATs (Appelbaum \& Grisso, 1995; Grisso \& Appelbaum, 1995; Grisso et al. 1995). Given that the data are consistently reported as highly skewed, a formal statistical comparison between the studies cannot be made, while study heterogeneity already renders comparison of questionable usefulness.

\section{Associations}

Most associations were reported as correlations with 'dimensional scores' based on the 'four factor model'. These are summarised and presented along with associations with the 'judgement standard' in Table 3. 
Table 2. Summary of DMC-T studies with a binary outcome of DMC-T

\begin{tabular}{|c|c|c|c|c|c|c|c|}
\hline Study & $\begin{array}{l}\text { DMC } \\
\text { standard }\end{array}$ & $\begin{array}{l}\text { Decision } \\
\text { assessed }\end{array}$ & Setting & Other relevant features & $\begin{array}{l}\text { Total } \\
\text { study } \\
N\end{array}$ & $\begin{array}{l}n \text { with } \\
\text { DMC-T }\end{array}$ & $\begin{array}{l}\text { Proportion with } \\
\text { DMC-T }(95 \% \mathrm{CI})\end{array}$ \\
\hline $\begin{array}{l}\text { Weinstock } \\
\text { et al. (1984) }\end{array}$ & Clinical & $\begin{array}{l}\text { Unrelated } \\
\text { medical } \\
\text { treatment }\end{array}$ & N/A & $\begin{array}{l}\text { Medically unwell in a } \\
\text { physical health hospital } \\
\text { referred for determination } \\
\text { of DMC-T for medical } \\
\text { treatment }\end{array}$ & $N=2$ & $n=2$ & $1(0.34-1)$ \\
\hline $\begin{array}{c}\text { Veliz \& } \\
\text { James } \\
(1987)\end{array}$ & Clinical & $\begin{array}{l}\text { Related } \\
\text { psychiatric } \\
\text { treatment }\end{array}$ & Inpatients & $\begin{array}{l}\text { Referred to the Court for } \\
\text { determination of lack of } \\
\text { competency to refuse or } \\
\text { consent to treatment } \\
\text { forensic population }\end{array}$ & $N=35$ & $n=4$ & $0.11(0.05-0.26)$ \\
\hline $\begin{array}{c}\text { Bean et al. } \\
(1994)\end{array}$ & Clinical & $\begin{array}{l}\text { Related } \\
\text { psychiatric } \\
\text { treatment }\end{array}$ & Inpatients & Inpatients requiring ECT & $N=32$ & $n=19$ & $0.59(0.42-0.75)$ \\
\hline $\begin{array}{l}\text { Wong et al. } \\
(2000)\end{array}$ & Clinical & $\begin{array}{l}\text { Blood test - } \\
\text { unclear } \\
\text { degree } \\
\text { related }\end{array}$ & Mixed & & $N=21$ & $n=19$ & $0.90(0.71-0.97)$ \\
\hline $\begin{array}{l}\text { Bellhouse } \\
\text { et al. (2003) }\end{array}$ & Clinical & $\begin{array}{l}\text { Related } \\
\text { psychiatric } \\
\text { treatment }\end{array}$ & Inpatients & & $N=9$ & $n=6$ & $0.67(0.35-0.88)$ \\
\hline $\begin{array}{l}\text { Vollmann } \\
\text { et al. (2003) }\end{array}$ & Clinical & $\begin{array}{l}\text { Related } \\
\text { psychiatric } \\
\text { treatment }\end{array}$ & Inpatients & No detained patients. & $N=43$ & $n=35$ & $0.81(0.67-0.90)$ \\
\hline $\begin{array}{l}\text { Moye et al. } \\
(2008)\end{array}$ & Threshold & $\begin{array}{l}\text { Unrelated } \\
\text { medical } \\
\text { treatment }\end{array}$ & Outpatients & $>60$ years old & $N=20$ & $n=4$ & $0.2(0.08-0.42)$ \\
\hline $\begin{array}{l}\text { Cairns et al. } \\
(2005)\end{array}$ & Clinical & $\begin{array}{l}\text { Related } \\
\text { psychiatric } \\
\text { treatment }\end{array}$ & Inpatients & & $N=62$ & $n=30$ & $0.48(0.36-0.61)$ \\
\hline $\begin{array}{l}\text { Owen et al. } \\
(2009 a \\
2011)\end{array}$ & Clinical & $\begin{array}{l}\text { Related } \\
\text { psychiatric } \\
\text { treatment }\end{array}$ & Inpatients & & $N=93$ & $n=24$ & $0.26(0.18-0.36)$ \\
\hline $\begin{array}{l}\text { Di \& Cheng } \\
\text { (2013) }\end{array}$ & Threshold & $\begin{array}{l}\text { Related } \\
\text { psychiatric } \\
\text { treatment }\end{array}$ & Inpatients & $\begin{array}{l}\text { Guardian also needed to } \\
\text { agree in order to } \\
\text { participate in study. }\end{array}$ & $N=192$ & $n=138$ & $0.72(0.65-0.78)$ \\
\hline $\begin{array}{l}\text { Skipworth } \\
\text { et al. (2013) }\end{array}$ & Clinical & $\begin{array}{l}\text { Related } \\
\text { psychiatric } \\
\text { treatment }\end{array}$ & Mixed & $\begin{array}{l}\text { Mixed inpatients and } \\
\text { outpatients under forensic } \\
\text { services }\end{array}$ & $N=97$ & $n=63$ & $0.65(0.55-0.74)$ \\
\hline
\end{tabular}

\section{Associations with DMC-T}

With the exception of insight, neurocognition, and socio-economic status (which includes a measure of years of education) most studies found no associations with DMC-T measured using either 'dimensional scores' or the 'judgement standard'. There was no heterogeneity between direction of associations when they were found by studies.

There was strong evidence for a negative association between lack of insight and DMC-T (medium to large ES), and positive association between better neurocognitive performance and DMC-T (medium ES). These associations covered a range of different dimensions with no discernible pattern for individual abilities such as 'understanding'.

The lack of any association with most sociodemographic variables (age, gender, race) is notable. There was a positive association in one study with higher socio-economic status and DMC-T, and weak evidence for a positive association for more years of education and DMC-T, especially with 'Understanding' (small to large ES). 


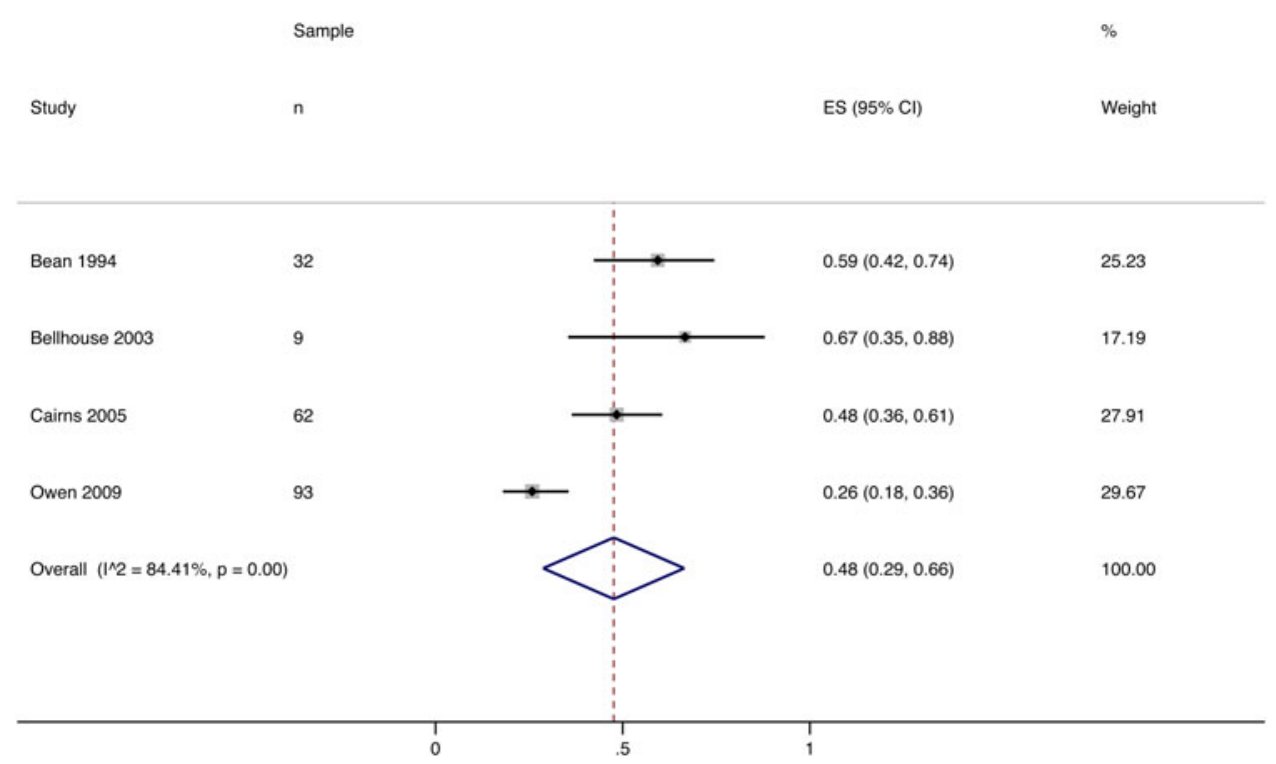

Fig. 2. Meta-analysis of proportions of patients with DMC-T.

With regards to symptoms of psychosis and DMC-T, there was some evidence for a negative association of PANSS total symptoms and PANSS negative symptoms with 'understanding' (medium to large ES). There was little evidence for a possible negative association of PANSS positive and PANSS general symptoms with dimension scores; overall, the majority of studies did not find any associations. One study reported on associations with BPRS factors. These are not included in the summary table (Appelbaum \& Grisso, 1995; Grisso \& Appelbaum, 1995; Grisso et al. 1995) but are in the online supplemental data table, and did not differ from the general pattern of the findings of associations of psychotic symptoms with DMC-T. No associations were found with affective symptoms.

\section{Associations with DMC-R}

The associations with DMC-R were similar to DMC-T with a few notable exceptions. Again, there was no heterogeneity between direction of associations when they were found by studies. As with DMC-T, other than one multi-centre study (Stroup et al. 2005), which reported negative associations between DMC-R and both 'nonwhite' ethnicity (small ES) and age and 'reasoning' (small ES), all studies found no associations with sociodemographics and DMC-R. Again there was weak evidence for a positive association for more years of education and DMC-R (small to large ES).

There was evidence for a positive association of better neurocognitive performance and DMC-R, which was much stronger than for DMC-T (small to large ES). By contrast, the associations with insight and
DMC-R were fewer and of smaller ES than with DMC-T (small to medium ES).

There was a range of negative associations with DMC-R and measures of psychotic symptoms (PANSS scores and BPRS - small to large ES), which appears stronger than with DMC-T, and perhaps not as specific to 'understanding'. Unlike DMC-T, there was also evidence for a negative association between PANSS general and PANSS negative symptoms with dimension scores. Two studies reported on associations with BPRS factors (again not included in the summary table but are included in the online supplemental data table) (Carpenter et al. 2000; Kovnick et al. 2003). These results did not substantially differ from the general pattern of the findings of associations of psychotic symptoms with DMC-R.

\section{Discussion}

\section{DMC-T v. DMC-R in schizophrenia}

Following meta-analysis, DMC-T, when measured by the 'judgement standard' was present in $48 \%$ of people receiving inpatient treatment for schizophrenia. The range of the proportion with DMC-T was wide (26$67 \%$ ). Heterogeneity between both samples and different decisions for which DMC was assessed was high. Outside of the analysis of DMC-T restricted to inpatient populations, it is difficult to draw any other distinct conclusions, using either 'judgement standards' or 'cut-off standards', beyond the finding that there is a wide range of DMC-T and DMC-R proportions in different samples of people with schizophrenia. 
Table 3. Associations with DMC-T and DMC-R Associations with DMC-T in red, DMC-R in black

\begin{tabular}{|c|c|c|c|c|c|c|c|}
\hline \multirow[b]{2}{*}{ Effect size (cohen's d) } & \multicolumn{3}{|c|}{$\begin{array}{l}\text { Associated with lack of DMC/worse } \\
\text { performance on dimension scores }\end{array}$} & \multirow[b]{2}{*}{ No association } & \multicolumn{3}{|c|}{$\begin{array}{l}\text { Associated with presence of } \mathrm{DMC} / \text { better } \\
\text { performance on dimension scores }\end{array}$} \\
\hline & $\mathrm{L}$ & M & S & & S & M & $\mathrm{L}$ \\
\hline & $\geqslant 0.5$ & $<0.5$ & $<0.3$ & 0 & $<0.3$ & $<0.5$ & $\geqslant 0.5$ \\
\hline Lack of insight & P A R & $\begin{array}{l}\text { U A R C } \\
P^{\mathrm{a}}\end{array}$ & U A R & $\begin{array}{c}\text { U } \\
\text { U A R CC }\end{array}$ & & & \\
\hline PANSS Total & UU & $\begin{array}{l}\text { U A R } \\
\text { UU A }\end{array}$ & $\mathbf{R}$ & $\begin{array}{l}\text { AA RR CC } \\
\text { U A R CC }\end{array}$ & & & \\
\hline PANSS General & $\begin{array}{l}\text { U } \\
\text { U A R }\end{array}$ & U A C & UU A R C & $\begin{array}{l}\text { U AA RR CC } \\
\text { P }^{\mathrm{a}} \text { UU A RR C }\end{array}$ & & & \\
\hline PANSS +VE & & $\begin{array}{c}\mathrm{U} \\
\mathrm{A} R\end{array}$ & U A & $\begin{array}{c}\text { UUU AAAA RRRR CCC } \\
\mathbf{P}^{\mathrm{a}} \text { UUUUUU AAA RRRR CCCC }\end{array}$ & & & \\
\hline PANSS -VE & $\begin{array}{c}\mathrm{U} \\
\mathrm{UA}\end{array}$ & $\begin{array}{l}\text { UU } \\
\text { U A R }\end{array}$ & UU A R C & $\begin{array}{l}\text { U AAAA RRRR CCC } \\
\text { Pa UUU AA RRR CCC }^{\text {a }}\end{array}$ & & & \\
\hline BPRS & U A & $\begin{array}{c}P U \\
R\end{array}$ & & $\begin{array}{l}\text { UU AAA RRR CC } \\
\text { P U A R C }\end{array}$ & & & \\
\hline Affective symptoms & & & & $\begin{array}{l}\text { UUU AAA RRR CC } \\
P^{\mathrm{a}} \text { UU A R C }\end{array}$ & & & \\
\hline Higher neurocognitive performance & & & & $\begin{array}{c}\text { AA } \\
\text { P AA RRRR CCCCC }\end{array}$ & U A RR & $\begin{array}{c}\text { UU RR C } \\
\mathbf{P}^{\mathrm{b}} \mathbf{P}^{\mathrm{a}} \text { UUU AA }\end{array}$ & UUUUU AAA RR \\
\hline Older age & & & $\mathbf{R}$ & $\begin{array}{c}\text { PP UUUUU AAAAA RRRRR CCCC } \\
\text { Pa }^{\mathbf{a}} \text { UUUUUU AAAA RRR CCC }\end{array}$ & & & \\
\hline Male gender & & & & $\begin{array}{l}\text { PP UU AA RR CC } \\
\text { UUU AA RR C }\end{array}$ & & & \\
\hline Black and minority ethnicity & & & A R & $\begin{array}{l}\text { UU AA RR CC } \\
\text { UU A R C }\end{array}$ & & & \\
\hline Higher socio-economic status & & & & A & $\mathrm{R}$ & $\mathrm{U}$ & \\
\hline More years of education & & & & $\begin{array}{c}\text { P UU AAAAA RRRR CCCC } \\
P^{\mathrm{a}} \text { UUU A RR CCC }\end{array}$ & $\begin{array}{c}\mathrm{R} \\
\mathrm{U} A \mathrm{~A} R\end{array}$ & $\begin{array}{l}\text { UU } \\
\text { UA }\end{array}$ & $\begin{array}{l}\text { P U } \\
\text { A }\end{array}$ \\
\hline
\end{tabular}

Each letters symbolises an individual study finding an association, with horizontal position on the table representing direction of association and effect size. Individual letters represent the DMC standard the association was found with: P, association with binary outcome of DMC; U, association with 'understanding'; A, association with 'appreciation'; $\mathrm{R}$, association with 'reasoning'; $\mathrm{C}$, association with 'expressing a choice'.

${ }^{a}$ Dunn et al. (2007) used three standards as their binary outcome so the 'most' standard was selected as this required scoring in 'understanding', 'appreciation', and 'reasoning', rather than the other two standards, which just required scores in 'understanding'. Dunn also used two presented data on two summary summary neurocognitive scores (DRS and a neurocognitive $\mathrm{z}$ score), the neurocognitive $\mathrm{z}$ score is presented here.

${ }^{\mathrm{b}}$ Linder et al. (2012) presented data on two summary neurocognitive scores (FAB positive association of medium ES, ACE no association), the FAB score is reported here. 
There was little evidence that socio-demographic factors had an impact on DMC-T or DMC-R. The lack of association between DMC and basic demographics is both a reassuring and an important finding, given that DMC measurement outcomes should not, in principle, be influenced by age, gender, or ethnicity. It runs counter to common misconceptions or presumptions that might be made regarding a lack of DMC with certain demographic characteristics such as age. Nevertheless, there was some weak evidence of an association with greater years of education.

While there was strong evidence of an association between greater insight and DMC-T, evidence of a similar association with DMC-R was much weaker. Insight is a clinical concept, which does not feature explicitly in the legal tests for DMC (although it is arguably subsumed within 'appreciation'). The relation between insight and DMC poses particular conceptual difficulties because (Owen et al. 2009b) a key component of a person's autonomy is the right to refuse treatment when one has DMC. In effect, this means that the individual, whose decision-making is unimpaired, has the right for their disagreement with their clinician concerning the nature or treatment of their illness to be respected. Yet lack of insight is a clinical phenomenon, which comprises non-acknowledgement of illness (David, 1990), due to a specific pathological process of the illness itself, and which often manifests itself as treatment refusal. A judgement as to whether treatment refusal stems from the values and beliefs of someone with DMC or from lack of insight depends, primarily, on the judgement of the clinician (Owen et al. 2009b). In the context of a person with a severe mental illness who is refusing treatment, there are understandable legal concerns if treatment refusal is equated with lack of DMC-T. At the same time, lack of insight is a common and core element of psychosis (David, 1990), which can, as our review demonstrates, have a substantial impact on DMC. These conceptual complexities are a natural corollary of mapping a medico-legal test onto clinical concepts.

The finding of associations between total symptoms (measured as PANSS total score or BPRS), negative symptoms and dimension scores is as we might expect, although it is curious that evidence is less convincing for DMC-T than DMC-R. The lack of association between positive symptoms and dimension scores in DMC-T and DMC-R is an interesting finding, which runs counter to anecdotal clinical experience and requires further investigation. These findings may be due to few participants with severe positive symptoms of psychosis being recruited for studies - many studies systematically excluded severely unwell people, either directly (through requiring vetting from the treating clinician), or indirectly (through recruiting in stable outpatient settings or setting a threshold of understanding or DMC for involvement in the primary study itself). Another possibility is that severe positive symptoms themselves (such as persecutory delusional beliefs) may result in participation refusal.

Given that studies investigating DMC are vulnerable to this selection bias, we consider it important that studies are designed to recruit from homogenous settings or disorders and minimise selection bias for participants with severe illness or lacking DMC-R for the study itself. A few studies have tackled this by collecting data on non-participants (Cairns et al. 2005; Owen et al. 2009a, 2011; Skipworth et al. 2013), but none have presented data on the symptom profile of nonparticipants in order to investigate further the lack of reported associations with DMC and positive symptoms.

There was evidence that better neurocognitive performance was positively associated with DMC-T. The evidence for this association in DMC-R was stronger, where better neurocognitive performance was highly positively associated with 'understanding' and, to a lesser extent, with 'appreciation' and 'reasoning'. This could suggest that a decision about participation in research presents a greater cognitive burden than DMC-T. If this is the case, it has implications for how information should be presented to potential participants. There is already evidence that educational (Dunn et al. 2002) and multimedia interventions (Jeste et al. 2009) can improve DMC-R in people with psychosis, mainly through enhancing 'understanding'. An alternative possibility is that, whereas a DMC-R testing paradigm is likely to present new information, within a DMC-T study, 'understanding' may already have been supported through treatment discussions in years of clinical interactions.

\section{Methodological limitations}

Sample size between studies varied considerably, with the exception of one outlier study with $n=1447$, the range was $n=2-192$ with a median of 37.5 , interquartile range 42 . The majority of studies did not provide information on sampling frames and recruitment rates. Although some provided information on nonparticipants (Cairns et al. 2005; Owen et al. 2009a 2011; Skipworth et al. 2013), this was for people of all diagnoses and hence could not be used specifically to refer to people with schizophrenia.

Inappropriate statistical analyses were often employed in source publications. Within the DMC-T studies there were many studies with substantial biases or study specific features, such as the assessment of DMC-T for unrelated medical treatment or the restriction of sampling to those referred for a 
secondary opinion of DMC-T or those refusing treatment (see Table 1 and online supplemental data table).

The review was limited by significant heterogeneity between studies, with differences between the outcome tools used, the decisions in relation to which DMC was assessed and the sampled populations. For the analysis of DMC proportions, such differences were managed through stratifications using narrow inclusion criteria. For the analysis of factors associated with DMC, given the extensive differences between all studies, stratification of analysis was not possible and all studies were therefore considered. Accordingly, due to possible confounders, we would recommend that these results are interpreted with caution.

The decision-specificity of DMC is an important source of the heterogeneity within the literature. Even for clearly defined decisions around, for example, treatment for schizophrenia, the precise nature of the decision, such as Electro-Convulsive Therapy $v$. antipsychotic treatment with clozapine, may lend itself to different vulnerabilities in the different abilities that make up DMC. While cognitively demanding decisions may require better performance on 'understanding' and 'reasoning', there is limited ability to compare the dimensional measures accordingly between studies.

The nature of the decision in relation to which DMC-R was tested requires special comment. It is important to point out that many of the DMC-R studies tested decisions relating to research which could not be considered as schizophrenia-specific, but which concerned a generic treatment, aimed at a general population. Several tested DMC-R concerning a trial of an experimental drug, which may help cognitive deficits, both in schizophrenia and in normal ageing. This decision, therefore, related to non schizophrenia-specific therapeutic research, where the salience of the decision to their present symptoms would vary substantially between participants and where the role of insight and other factors was unclear and not homogenous. The contribution of these studies to understanding DMC-R in schizophrenia in relation to therapeutic research for schizophrenia is thus unclear. Decisions around research participation for therapeutic or non-therapeutic research may also pose different challenges, given the different risk/ benefit profiles for the individual, and may therefore further complicate direct comparison between studies.

As a consequence there remains a need to unpick, which what abilities are global, impacting decisionmaking in general, and which are specific to the particular decision in hand. We hypothesise that lack of insight into one's illness would be relatively circumscribed to decisions around treatment or life consequences of the functional deficits of the illness through impact on 'appreciation', compared with symptoms such as 'thought disorder', which may affect decision making more generally through impact on 'understanding'.

The effect of publication bias on this review is unclear. Funnel plots are difficult to do with this data but as most studies report simple proportions and/or multiple association analysis there are no strong reasons to suspect publication bias.

\section{Categorical v. dimensional measures of DMC}

The majority of studies we found used 'dimensional scores' for their measurements of DMC. The 'judgment standard' when used, was used in isolation or guided by tools using 'dimensional scores'.

Dimensional measures of DMC take an overly siloed view of the DMC construct, and it is likely these abilities are not independent of each other. It is clear from our work that poor performance on different individual measures can impact others (if there are profound deficits on 'understanding', then there will be resultant deficits on 'appreciation' or 'use or weigh'; conversely in people with low insight this can be a total barrier to discussing the nature of their illness, even in abstract, and result in serious doubts about their resultant 'understanding'). This creates a hierarchical element to dimensional measures of $\mathrm{DMC}$, in that sufficient performance on one ability is pre-requisite to performance on other abilities.

Dimensional measures can in some situations be relatively insensitive to deficits that categorical measures can detect. Some elements of psychopathology can be highly circumscribed, and have marked impact on DMC as measured by a categorical standard, but relatively less impact on dimensional measures. For example, an isolated delusional belief that participation within a research study will cure the participant of all illness may result in partially reduced scores on 'appreciation' and 'reasoning' when assessed using the framework of the MacCAT-CR, but a clear lack of DMC-R when using a 'judgement standard'. Given the limitations to using dimensional measures in isolation, we recommend that future research employ both dimensional and judgement measures of DMC.

\section{Conclusions}

We found that a significant proportion of people with schizophrenia, even on inpatient wards, have DMC, that DMC is associated with clinically relevant variables, such as insight and neurocognitive performance, and that DMC is not related to socio-demographic factors. 
There have been many studies investigating DMC in schizophrenia in the past two decades. To our knowledge, this is the most methodologically rigorous attempt to synthesise the findings from these studies, and one that was not limited to one standard of assessment of DMC or one type of decision for which DMC was assessed such as DMC-T or DMC-R. This review is the first to overview the field, and draws broad conclusions regarding the proportion and associations of DMC in schizophrenia and compare and contrast these for DMC-T and DMC-R. It is clear, however, that the complexity of the DMC construct resulting from its decision-specificity and the dimensional and categorical approaches to measuring it renders the literature diverse. Arguably it is in disarray. In order to develop our understanding of DMC in schizophrenia future quantitative research should involve comparative studies of DMC, using both dimensional and categorical measures, and provide data on nonparticipants and sampling-frames. Otherwise the time and decision-specific nature of DMC may lead to study-specificity, which renders systematic review impossible.

\section{Acknowledgement}

This report is independent research arising from a Doctoral Research Fellowship held by B Spencer, DRF-2014-07-035 supported by the National Institute for Health Research. The views expressed in this publication are those of the author(s) and not necessarily those of the NHS, the National Institute for Health Research or the Department of Health.

\section{Supplementary material}

For supplementary material accompanying this paper visit https://doi.org/10.1017/S0033291717000502.

\section{References}

American Psychiatric Association (1994). Diagnostic and Statistical Manual of Mental Disorders: DSM-IV. American Psychiatric Association: Washington, DC.

Appelbaum PS (2007). Clinical practice. Assessment of patients' competence to consent to treatment. New England Journal of Medicine 357, 1834-1840.

Appelbaum PS, Grisso T (1995). The MacArthur Treatment Competence Study. I: mental illness and competence to consent to treatment. Law and Human Behavior 19, 105-126.

Appelbaum PS, Grisso T (2001). MacCAT-CR MacArthur Competence Assessment Tool for Clinical Research. Professional Resource Press: Sarasota, Florida.

Barrett JJ, Hart KJ, Schmerler JT, Willmarth K, Carey JA, Mohammed S (2009). Criterion validity of the financial skills subscale of the direct assessment of functional status scale. Psychiatry Research 166, 148-157.

Bean G, Nishisato S, Rector NA, Glancy G (1994). The psychometric properties of the Competency Interview Schedule. Canadian Journal of Psychiatry 39, 368-376.

Bellhouse J, Holland AJ, Clare ICH, Gunn M, Watson P (2003). Capacity-based mental health legislation and its impact on clinical practice: 2) treatment in hospital. Journal of Mental Health Law 37, 24-28.

Cairns R, Maddock C, Buchanan A, David AS, Hayward P, Richardson G, Szmukler G, Hotopf M (2005). Prevalence and predictors of mental incapacity in psychiatric in-patients. British Journal of Psychiatry 187, 379-385.

Candilis PJ, Fletcher KE, Geppert CM, Lidz CW, Appelbaum PS (2008). A direct comparison of research decision-making capacity: schizophrenia/schizoaffective, medically ill, and non-ill subjects. Schizophrenia Research 99, 350-358.

Candilis PJ, Geppert CM, Fletcher KE, Lidz CW, Appelbaum PS (2006). Willingness of subjects with thought disorder to participate in research. Schizophrenia Bulletin 32, 159-165.

Capdevielle D, Raffard S, Bayard S, Garcia F, Baciu O, Bouzigues I, Boulenger J-P (2009). Competence to consent and insight in schizophrenia: is there an association? A pilot study. Schizophrenia Research 108, 272-279.

Carpenter Jr WT, Gold JM, Lahti AC, Queern CA, Conley RR, Bartko JJ, Kovnick J, Appelbaum PS (2000). Decisional capacity for informed consent in schizophrenia research. Archives of General Psychiatry 57, 533-538.

Chiu NM, Lee Y, Lee WK (2014). Electroconvulsive therapy without consent from patients: one-year follow-up study. Asia-Pacific Psychiatry 6, 83-90.

Cohen BJ, McGarvey EL, Pinkerton RC, Kryzhanivska L (2004). Willingness and competence of depressed and schizophrenic inpatients to consent to research. Journal of the American Academy of Psychiatry and the Law 32, 134-143.

Cohen J (1992). A power primer. Psychological Bulletin 112, 155-159.

David AS (1990). Insight and psychosis. British Journal of Psychiatry 156, 798-808.

De Marco MC, Sani G, Manfredi G, Pacchiarotti I, Savoja V, Balbi A, Mazzarini L, Borriello A, Kotzalidis GD, Tatarelli R, Girardi P, Ferracuti S (2010). Assessment of the capacity to express informed consent for organ donation in patients with schizophrenia. Journal of Forensic Sciences 55, 669-676.

Di X, Cheng HG (2013). Competence of consent and associated factors among inpatients of schizophrenia in Changsha, China. Schizophrenia Research 150, 325-326.

Dunn LB, Lindamer LA, Palmer BW, Golshan S, Schneiderman LJ, Jeste DV (2002). Improving understanding of research consent in middle-aged and elderly patients with psychotic disorders. American Journal of Geriatric Psychiatry 10, 142-150.

Dunn LB, Palmer BW, Appelbaum PS, Saks ER, Aarons GA, Jeste DV (2007). Prevalence and correlates of adequate performance on a measure of abilities related to decisional capacity: differences among three standards for the 
MacCAT-CR in patients with schizophrenia. Schizophrenia Research 89, 110-118.

Eyler LT, Mirzakhanian H, Jeste DV (2005). A preliminary study of interactive questioning methods to assess and improve understanding of informed consent among patients with schizophrenia. Schizophrenia Research 75, 193-198.

Eyler LT, Olsen RK, Nayak GV, Mirzakhanian H, Brown GG, Jeste DV (2007). Brain response correlates of decisional capacity in schizophrenia: a preliminary fMRI study. Journal of Neuropsychiatry and Clinical Neurosciences 19, 137-144.

Fischer BA, McMahon RP, Meyer WA, Slack DJ, Appelbaum PS, Carpenter WT (2013). Participants with schizophrenia retain the information necessary for informed consent during clinical trials. Journal of Clinical Psychiatry 74, 622-627.

Grisso T, Appelbaum PS (1995). The MacArthur Treatment Competence Study. III: abilities of patients to consent to psychiatric and medical treatments. Law and Human Behavior 19, 149-174.

Grisso T, Appelbaum PS (1998). Assessing Competence to Consent to Treatment: A Guide for Physicians and other Health Professionals. Oxford University Press: New York.

Grisso T, Appelbaum PS, Hill-Fotouhi C (1997). The MacCAT-T: a clinical tool to assess patients' capacities to make treatment decisions. Psychiatric Services 48, 1415-1419.

Grisso T, Appelbaum PS, Mulvey EP, Fletcher K (1995). The MacArthur Treatment Competence Study. II: measures of abilities related to competence to consent to treatment. Law and Human Behavior 19, 127-148.

Jacob R, Gunn M, Holland A eds. (2013). Mental Capacity Legislation: Principles and Practice. RCPsych Publications: London.

Jeste DV, Depp CA, Palmer BW (2006). Magnitude of impairment in decisional capacity in people with schizophrenia compared to normal subjects: an overview. Schizophrenia Bulletin 32, 121-128.

Jeste DV, Palmer BW, Golshan S, Eyler LT, Dunn LB, Meeks T, Glorioso D, Fellows I, Kraemer H, Appelbaum PS (2009). Multimedia consent for research in people with schizophrenia and normal subjects: a randomized controlled trial. Schizophrenia Bulletin 35, 719-729.

Kavanagh A, Browne S, Horgan R, O'Sullivan D, Sheppard N, Kirby M (2008). Ireland's long-stay charge refunds: mental capacity and dilemmas in a psychiatric institution. Irish Journal of Psychological Medicine 25, 100-103.

Kay SR, Fiszbein A, Opler LA (1987). The positive and negative syndrome scale (PANSS) for schizophrenia. Schizophrenia Bulletin 13, 261-276.

Koren D, Poyurovsky M, Seidman LJ, Goldsmith M, Wenger S, Klein EM (2005). The neuropsychological basis of competence to consent in first-episode Schizophrenia: a pilot Metacognitive Study. Biological Psychiatry 57, 609-616. Kovnick JA, Appelbaum PS, Hoge SK, Leadbetter RA (2003). Competence to consent to research among long-stay inpatients with chronic schizophrenia. Psychiatric Services 54, 1247-1252.

Kumar TC, John S, Gopal S, Mohan G, Joseph J, Rangaswamy T, PAS Study Group (2013). Psychiatric advance statements: an Indian experience. International Journal of Social Psychiatry 59, 531-534.

Lan T-H, Wu B-J, Chen H-K, Liao H-Y, Lee S-M, Sun H-J (2013). Validation of Chinese version of the MacArthur Competence Assessment Tool for Clinical Research (MacCAT-CR) in patients with schizophrenia spectrum disorders. Psychiatry Research 210, 634-640.

Linder M, Ari LL, Kurs R, Melamed Y (2012). Evaluation of the capacity of inpatients with chronic schizophrenia to provide informed consent for participation in clinical trials: use of the hebrew version of the MacArthur competence assessment tool for clinical research (MacCAT-CR). Israel Medical Association Journal 14, 470-474.

Moser DJ, Reese RL, Hey CT, Schultz SK, Arndt S, Beglinger LJ, Duff KM, Andreasen NC (2006). Using a brief intervention to improve decisional capacity in schizophrenia research. Schizophrenia Bulletin 32, 116-120.

Moser DJ, Reese RL, Schultz SK, Benjamin ML, Arndt S, Fleming FW, Andreasen NC (2005). Informed consent in medication-free Schizophrenia Research. American Journal of Psychiatry 162, 1209-1211.

Moser DJ, Schultz SK, Arndt S, Benjamin ML, Fleming FW, Brems CS, Paulsen JS, Appelbaum PS, Andreasen NC (2002). Capacity to provide informed consent for participation in schizophrenia and HIV research. American Journal of Psychiatry 159, 1201-1207.

Moye J, Karel MJ, Edelstein B, Hicken B, Armesto JC, Gurrera RJ (2008). Assessment of capacity to consent to treatment: challenges, the "ACCT" approach, future directions. Clinical Gerontologist: Journal of Aging and Mental Health 31, 37-66.

Nicholson TR, Cutter W, Hotopf M (2008). Assessing mental capacity: the Mental Capacity Act. British Medical Journal 336, 322-325.

Norko M, Billick SB, McCarrick RG, Schwartz MA (1990). A clinical study of competency to consent to voluntary psychiatric hospitalization. American Journal of Forensic Psychiatry 11, 3-15.

Overall JE, Gorham DR (1962). The brief psychiatric rating scale. Psychological Reports 10, 79-812.

Owen GS, David AS, Richardson G, Szmukler G, Hayward P, Hotopf M (2009a). Mental capacity, diagnosis and insight in psychiatric in-patients: a cross-sectional study. Psychological Medicine 39, 1389-1398.

Owen GS, Freyenhagen F, Richardson G, Hotopf M (2009b). Mental capacity and decisional autonomy: an interdisciplinary challenge. Inquiry 52, 79-107.

Owen GS, Ster IC, David AS, Szmukler G, Hayward P, Richardson G, Hotopf M (2011). Regaining mental capacity for treatment decisions following psychiatric admission: a clinico-ethical study. Psychological Medicine 41, 119-128.

Palmer BW, Dunn LB, Appelbaum PS, Jeste DV (2004). Correlates of treatment-related decision-making capacity among middle-aged and older patients with Schizophrenia. Archives of General Psychiatry 61, 230-236.

Palmer BW, Dunn LB, Appelbaum PS, Mudaliar S, Thal L, Henry R, Golshan S, Jeste DV (2005). Assessment of capacity to consent to research among older persons with 
Schizophrenia, Alzheimer disease, or diabetes Mellitus: comparison of a 3-item questionnaire with a comprehensive standardized capacity instrument. Archives of General Psychiatry 62, 726-733.

Palmer BW, Jeste DV (2006). Relationship of individual cognitive abilities to specific components of decisional capacity among middle-aged and older patients with schizophrenia. Schizophrenia Bulletin 32, 98-106.

Raffard S, Fond G, Brittner M, Bortolon C, Macgregor A, Boulenger J-P, Gely-Nargeot MC, Capdevielle D (2013). Cognitive insight as an indicator of competence to consent to treatment in schizophrenia. Schizophrenia Research 144, 118-121.

Skipworth JJ, Dawson J, Ellis PM (2013). Capacity of forensic patients to consent to treatment. Australian and New Zealand Journal of Psychiatry 47, 443-450.

Srebnik D, Appelbaum PS, Russo J (2004). Assessing competence to complete psychiatric advance directives with the competence assessment tool for psychiatric advance directives. Comprehensive Psychiatry 45, 239-245.

Stroup S, Appelbaum P, Swartz M, Patel M, Davis S, Jeste D, Kim S, Keefe R, Manschreck T, McEvoy J, Lieberman J (2005). Decision-making capacity for research participation among individuals in the CATIE schizophrenia trial. Schizophrenia Research 80, 1-8.

Valletto NM, Kamahele R, Menon AS, Ruskin P (2002). Completion of advance directives for general health care among inpatients with schizophrenia. Journal of Nervous and Mental Disease 190, 264-265.

Veliz J, James WS (1987). Medicine court: Rogers in practice. American Journal of Psychiatry 144, 62-67.

Vollmann J, Bauer A, Danker-Hopfe H, Helmchen H (2003). Competence of mentally ill patients: a comparative empirical study. Psychological Medicine 33, 1463-1471.

Wang SB, Wang YY, Ungvari GS, Ng CH, Wu RR, Wang J, Xiang YT (2016). The MacArthur competence assessment tools for assessing decision-making capacity in schizophrenia: a meta-analysis. Schizophrenia Research http://dx.doi.org/10.1016/j.schres.2016.11.020.

Wechsler D (1997). Wechsler Adult Intelligence Scale, 3rd edn. (WAIS-III) Psychological Corp; 1997: San Antonio, Texas.

Weinstock R, Copelan R, Bagheri A (1984). Competence to give informed consent for medical procedures. Bulletin of the American Academy of Psychiatry and the Law 12, 117-125.

Wong JG, Cheung EP, Chen EY (2005). Decision-making capacity of inpatients with schizophrenia in Hong Kong. Journal of Nervous and Mental Disease 193, 316-322.

Wong JG, Clare CH, Holland AJ, Watson PC, Gunn M (2000). The capacity of people with a 'mental disability' to make a health care decision. Psychological Medicine 30, 295-306.

World Health Organization (1993). The ICD-10 Classification of Mental and Behavioural Disorders: Diagnostic Criteria for Research. World Health Organization: Geneva. 